\title{
The impact of teaching on the duration of common urological operations
}

\author{
Blayne Welk, MD;,1,2 Jennifer Winick-Ng, MSc; ${ }^{-2}$ Andrew McClure, MSc,; Chris Vinden, MD, ;,2 Sumit Dave, MD; \\ Stephen Pautler, $M D^{\prime}$
}

${ }^{1}$ University of Western Ontario, London, ON, Canada; Inssitute for Clinical Evaluative Sciences, London, ON, Canada

Cite as: Can Urol Assoc J 2016;10(5-6):172-8. http://dx.doi.org/10.5489/cua|.3737

See related commentary on page 179.

Visit the online version of this article at www.cuaj.ca as of June 15 for supplemental tables.

\section{Abstract}

Introduction: The ability of academic (teaching) hospitals to offer the same level of efficiency as non-teaching hospitals in a publicly funded healthcare system is unknown. Our objective was to compare the operative duration of general urology procedures between teaching and non-teaching hospitals.

Methods: We used administrative data from the province of Ontario to conduct a retrospective cohort study of all adults who underwent a specified elective urology procedure (2002-2013). Primary outcome was duration of surgical procedure. Primary exposure was hospital type (academic or non-teaching). Negative binomial regression was used to adjust relative time estimates for age, comorbidity, obesity, anesthetic, and surgeon and hospital case volume. Results: 114225 procedures were included (circumcision $n=12$ 280; hydrocelectomy $n=7221$; open radical prostatectomy $n=22$ 951; transurethral prostatectomy $n=56$ 066; or mid-urethral sling $\mathrm{n}=15$ 707). These procedures were performed in an academic hospital in $14.8 \%, 13.3 \%, 28.6 \%, 17.1 \%$, and $21.3 \%$ of cases, respectively. The mean operative duration across all procedures was higher in academic centres; the additional operative time ranged from 8.3 minutes (circumcision) to 29.2 minutes (radical prostatectomy). In adjusted analysis, patients treated in academic hospitals were still found to have procedures that were significantly longer (by 10-21\%). These results were similar in sensitivity analyses that accounted for the potential effect of more complex patients being referred to tertiary academic centres.

Conclusions: Five common general urology operations take significantly longer to perform in academic hospitals. The reason for this may be due to the combined effect of teaching students and residents or due to inherent systematic inefficiencies within large academic hospitals.

\section{Introduction}

Canadians place a significant value on maintaining a highquality, publicly accessible healthcare system. However, sustaining this system is challenging, as healthcare costs are rising faster than inflation. ${ }^{1}$ The majority of Canadian hospitals are funded by an annual fixed global budget, which tends to promote rationing of health services, increased wait times, and provides no incentive for productivity and efficiency. ${ }^{2}$ These issues have led to the implementation of alternative funding models, such as activity-based funding ("bundled reimbursement" or "quality-based funding"). ${ }^{3}$ For surgeons, this model has significant implications: if a hospital receives a single fixed payment for a specific service (such as joint replacement), then the hospital and surgeon must provide this service efficiently and economically in order to avoid losing money and potentially not being able to offer this procedure.

This funding shift has significant potential implications for academic hospitals. While non-teaching-based hospitals exist primarily to deliver patient care, academic hospitals have the additional demands of clinical research and the training and education of medical and nursing students, allied health professionals, and postgraduate physicians (interns, residents, and fellows). The inclusion of medical residents in surgical procedures performed in the U.S. has been shown to increase the risk of a prolonged operation for specific urological procedures (such as laparoscopic urological oncology procedures ${ }^{4}$ and transurethral surgery ${ }^{5}$ ), as well as in other surgical specialities (such as gynecology ${ }^{6}$ and general surgery ${ }^{7}$ ). The increase in operative duration associated with training future medical professionals inherently increases the operating room cost of procedures at academic hospitals and is not currently accounted for in many activity-based funding models. Even small increases in operative time are significant, as each minute of operating room time has been calculated to cost between $\$ 10$ and $\$ 40$ in Canada. ${ }^{8}$ 
To our knowledge, the investigation of the actual magnitude of impact that medical education has on the operating room efficiency of urologists has not been quantified in a publically funded healthcare system. The primary objective of this study was to compare the operative time of five general urology procedures when they were performed in academic hospitals as compared to non-teaching hospitals.

\section{Methods}

\section{Study design and setting}

This study was conducted at the Institute for Clinical Evaluative Sciences (ICES). We performed a populationbased, retrospective cohort study of all adult patients who underwent one of five general urological procedures between April 2002 and March 2013 in the province of Ontario (population of approximately 13 million people, with universal healthcare). Study approval was granted through the Research Ethics Board at Sunnybrook Hospital (Toronto, ON).

\section{Data sources}

The Canadian Institute for Health Information's Discharge Abstract Database and Same Day Surgery (CIHI-DAD/SDS) databases capture all diagnostic and procedural information for patients who are admitted to hospital or who undergo an inpatient or outpatient surgical procedure. The Ontario Health Insurance Plan (OHIP) database captures all health claims for physician services. The ICES Physician Database (IPDB) contains physician characteristics. The Registered Persons Database (RPDB) captures demographic data on all individual Ontarians. These datasets were linked using unique, encoded identifiers and analyzed at the ICES Western site. Previous studies have demonstrated that these data sources are reliable and valid. ${ }^{9-11}$

\section{Patient population}

We identified patients who had both a relevant Canadian Classification of Health Intervention (CCl) code and a matching OHIP billing code for one of the following five procedures: circumcision, hydrocelectomy, open radical prostectomy, transurethral prostectomy, or mid-urethral sling. As the OHIP fee code for a mid-urethral sling was only introduced in the fall of 2007, we restricted the mid-urethral sling cohort to Oct 2007-March 2013.

Of the 189596 patients initially considered for inclusion, we excluded patients who had missing or invalid information (missing institution number, invalid demographic information, $n=440$ ), were treated at a pediatric hospital, or in the
Kingston area $(\mathrm{n}=2727$, due to historically inconsistent OHIP billing in that region), were $<18$ years of age ( $n=19206)$, or were from outside Ontario $(n=64)$. We also excluded emergency $(n=8753)$ and after-hours $(n=2578)$ procedures, repeat procedures (using a minimum 10-year lookback window, $\mathrm{n}=10$ 078), procedures performed by surgeons other than urologists or gynecologists $(n=1150)$, and those without an anesthesiologist present $(n=2974)$ or an anesthetic billing record from the same day $(n=9382)$. Patients who had a simultaneous additional procedure $(n=17$ 855) were also excluded; however, select, commonly occurring concurrent procedures were permitted and adjusted for in the multivariable analysis.

\section{Primary outcome and exposure}

The primary outcome was surgical duration. This was determined using a previously validated algorithm (with a correlation of $r=0.94$ with medical records), which calculates the duration of an operative procedure based on anesthesia OHIP billing records. ${ }^{12}$ Time units are billed in 15-minute increments and encompass the time period from which the patient enters the operating room to the time the patient is transferred to the post-surgical recovery unit. Our prespecified hypothesis was that surgery would take longer in academic centres.

The primary exposure was surgery carried out in an academic hospital (defined as a hospital with full-time medical school and residency training programs, and a primary affiliation with a medical school). This included specific hospitals in Hamilton (McMaster University), London (Western University), Ottawa (Ottawa University), and Toronto (University of Toronto). Kingston (Queens University) was excluded due to their alternative funding model for physicians.

\section{Covariates}

Measured patient covariates include age, socioeconomic status (based on income quintiles), rural residence, ${ }^{13}$ morbid obesity (body mass index $>45$ ), and number of family physician visits in the year prior to the procedure. Expected healthcare use (as a marker of comorbidity) was measured using the Johns Hopkins University Adjusted Case Groups ${ }^{\circledR}$ case-mix system Resource Utilization Bands (RUB); this system classifies all inpatient and outpatient healthcare visits based on disease severity and chronicity. ${ }^{14}$ RUBs were classified as low $(R \cup B=0-3)$, moderate $(R \cup B=4)$, or high $(R \cup B=5)$. Procedure covariates included year, type of anesthesia (general anesthesia (GA), spinal/epidural, other), and the yearly volume of the specific procedure at the hospital (determined from CIHI-DAD/SDS). Physician covariates from the IPDB included age, gender, specialty, and surgeon yearly procedure volume (calculated using OHIP records). 
Welk et al.

\section{Statistical analysis}

Mean and standard deviation are used to report our primary outcome and continuous variable covariates. Baseline characteristics of each procedure were compared between academic and non-teaching hospitals using standardized differences (SD). SD provide a better indication of clinically meaningful differences than $p$ values when study samples are large (SD greater than $10 \%$ is considered potentially significant). ${ }^{15}$

The primary analysis was a negative binomial regression model with generalized estimated equations to account for the shared variance of patients clustered within surgeons, who in turn were clustered within hospitals (SAS 9.3, SAS institute, Cary, NC, U.S.). Unadjusted and adjusted (for patient age, comorbidity score, morbid obesity, year of surgery, anesthesia type, physician age, gender, yearly hospital and surgeon volume, and specific concurrent procedures) models are presented and time ratios (representing the relative change in surgical duration), 95\% confidence intervals, and $p$ values are reported $(p<0.05$ was considered significant).

Two additional sensitivity analyses were conducted to assess for a referral bias. First, we restricted our analysis to patients with low comorbidity (RUB 0-3). Second, we restricted our analysis to patients who presented to their expected admitting hospital, based on data from the Ontario Multispecialty Network database. ${ }^{16}$

\section{Results}

We identified a total of 114225 people who met our study inclusion criteria (circumcision $n=12$ 280; hydrocelectomy $\mathrm{n}=7221$; open radical prostatectomy $\mathrm{n}=22$ 951; transurethral prostatectomy $n=56066$; or mid-urethral sling $n=15$ 707). These procedures were performed at an academic hospital in $14.8 \%, 13.3 \%, 28.6 \%, 17.1 \%$, and $21.3 \%$, respectively. The use of pelvic lymphadenectomy during radical prostatectomy was slightly more prevalent in non-teaching hospitals $(91.2 \%$ vs. $87.9 \%$, SD 0.11$)$. Among women receiving a mid-urethral sling, $57.6 \%$ were done by gynecologists and $42.4 \%$ were done by urologists. Selected baseline characteristics of the patients and surgeons involved in these procedures are shown in Table 1. In general, patient characteristics were similar between those undergoing procedures at an academic hospital compared to a non-teaching hospital, with a larger proportion of rural patients attending a non-teaching hospital. Patients at academic hospitals were more likely to have general anesthesia (GA) for a radical prostatectomy and less likely to have GA for a mid-urethral sling. Surgeons at academic hospitals tended to perform more radical prostatectomies and mid-urethral slings compared to those operating in non-teaching hospitals (who conversely performed more circumcisions, hydrocelectomies, and transurethral prostectomies). Academic hospitals tended to have a lower yearly volume of hydrocelectomies and circumcisions and a higher volume of mid-urethral slings and radical prostatectomies.

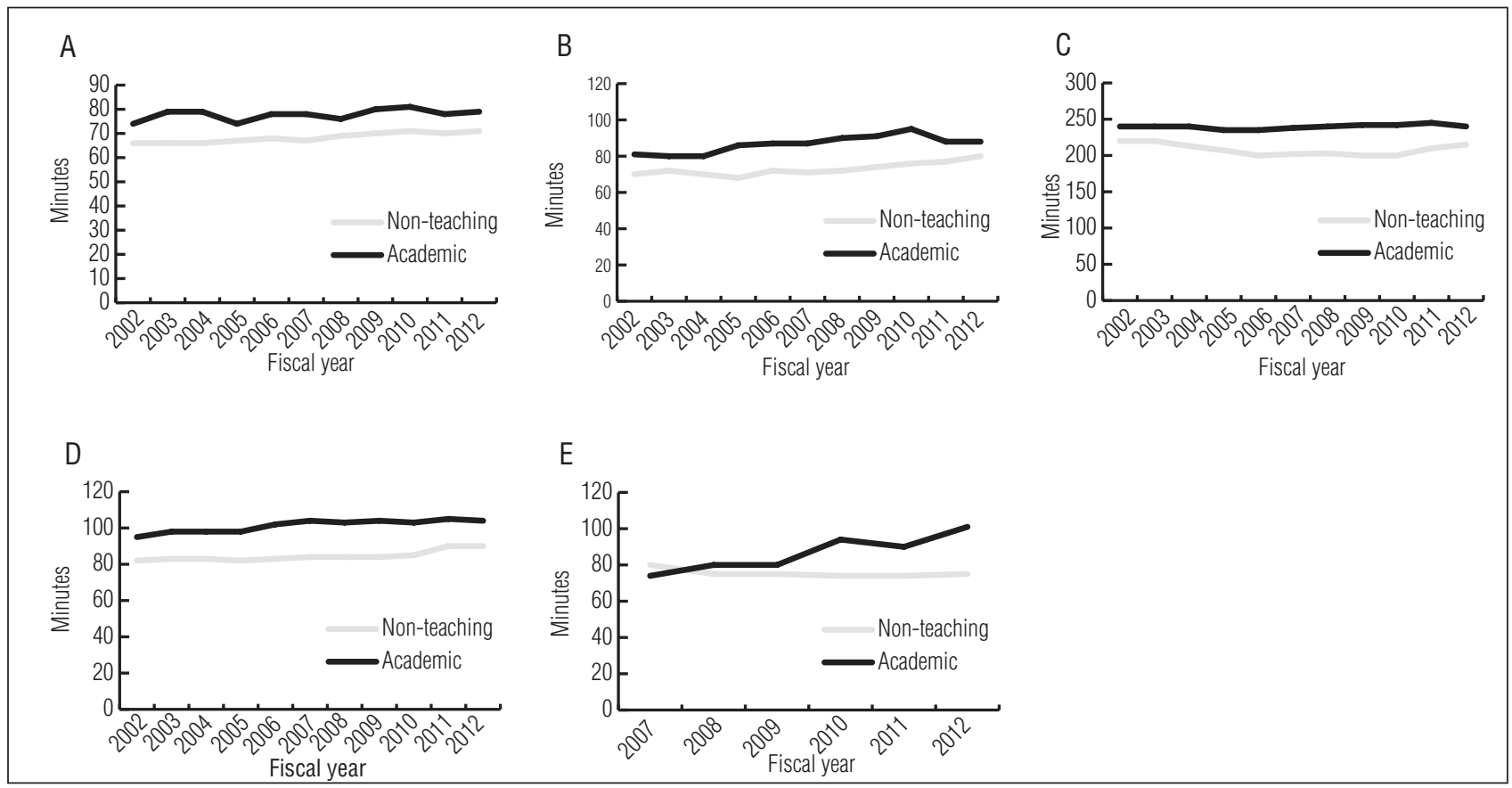

Fig. 1. Duration of general urological procedures by hospital type between 2002 and 2012: (A) Circumcision; (B) Hydrocelectomy; (C) Open radical prostatectomy; (D)Transurethral prostatectomy; (E) Mid-urethral sling. 
In all cases, the unadjusted operative time was longer for procedures done in academic hospitals compared to non-teaching hospitals (Fig. 1). The mean additional time (over the entire study period) that was required in academic hospitals to carry out these procedures was: circumcision 8.3 minutes (95\% Cl 7.2-9.4); hydrocelectomy $12.8 \mathrm{~min}-$ utes $(95 \% \mathrm{Cl} 11.0-14.6)$; radical prostatectomy $29.2 \mathrm{~min}-$ utes $(95 \% \mathrm{Cl} 27.6-30.8)$; transurethral prostatectomy 16.6 minutes (95\% Cl 15.9-17.4); and mid-urethral sling 14.1 minutes $(95 \% \mathrm{Cl} 12.6-15.6)$.

In our multivariable model, all five general urology pro- cedures continued to take significantly longer in academic hospitals compared to non-teaching hospitals (Table 2). The increased time required for these procedures in academic hospitals compared to non-teaching hospitals varied from $10-21 \%$ (Fig. 2A). Morbid obesity was associated with a prolonged operative time in all procedures. All procedures except radical prostatectomy were significantly longer with the use of epidural/spinal anesthesia and increased surgeon volume significantly reduced operative time for circumcision, hydrocelectomy, and radical prostatectomy. In our two preplanned sensitivity analyses, the significantly

\begin{tabular}{|c|c|c|c|c|c|c|c|c|c|c|}
\hline & \multicolumn{2}{|c|}{ Circumcision } & \multicolumn{2}{|c|}{ Hydrocelectomy } & \multicolumn{2}{|c|}{$\begin{array}{l}\text { Open radical } \\
\text { prostatectomy }\end{array}$} & \multicolumn{2}{|c|}{$\begin{array}{l}\text { Transurethral } \\
\text { prostatectomy }\end{array}$} & \multicolumn{2}{|c|}{ Mid-urethral sling } \\
\hline & $\begin{array}{c}\text { Non- } \\
\text { teaching }\end{array}$ & Academic & $\begin{array}{c}\text { Non- } \\
\text { teaching }\end{array}$ & Academic & $\begin{array}{c}\text { Non- } \\
\text { teaching }\end{array}$ & Academic & $\begin{array}{c}\text { Non- } \\
\text { teaching }\end{array}$ & Academic & $\begin{array}{c}\text { Non- } \\
\text { teaching }\end{array}$ & Academic \\
\hline $\begin{array}{l}\text { Number of } \\
\text { procedures (\%) }\end{array}$ & $\begin{array}{c}10461 \\
(85.2 \%)\end{array}$ & $\begin{array}{c}1819 \\
(14.8 \%)\end{array}$ & $\begin{array}{c}6257 \\
(86.7 \%)\end{array}$ & $\begin{array}{c}964 \\
(13.3 \%)\end{array}$ & $\begin{array}{c}16386 \\
(71.4 \%)\end{array}$ & $\begin{array}{c}6565 \\
(28.6 \%)\end{array}$ & $\begin{array}{c}46468 \\
(82.9 \%)\end{array}$ & $\begin{array}{c}9598 \\
(17.1 \%)\end{array}$ & $\begin{array}{c}12353 \\
(78.6 \%)\end{array}$ & $\begin{array}{c}3354 \\
(21.4 \%)\end{array}$ \\
\hline \multicolumn{11}{|c|}{ Patient characteristics } \\
\hline Age & $\begin{array}{c}47.5 \\
(20.4)\end{array}$ & $\begin{array}{c}45.5 \\
(19.9)^{*}\end{array}$ & $\begin{array}{c}54.8 \\
(16.1)\end{array}$ & $\begin{array}{c}54.8 \\
(15.9)\end{array}$ & $\begin{array}{l}62.0 \\
(6.4)\end{array}$ & $\begin{array}{l}61.6 \\
(6.6)\end{array}$ & $\begin{array}{l}71.5 \\
(9.2)\end{array}$ & $\begin{array}{l}70.8 \\
(9.3)\end{array}$ & $\begin{array}{c}53.1 \\
(11.8)\end{array}$ & $\begin{array}{c}53.5 \\
(11.2)\end{array}$ \\
\hline $\begin{array}{l}\text { Number of } \\
\text { primary care } \\
\text { visits }\end{array}$ & $\begin{array}{c}6.1 \\
(6.3)\end{array}$ & $\begin{array}{c}5.3 \\
(6.4)^{*}\end{array}$ & $\begin{array}{c}5.7 \\
(5.5)\end{array}$ & $\begin{array}{c}5.4 \\
(6.1)\end{array}$ & $\begin{array}{c}6.3 \\
(4.7)\end{array}$ & $\begin{array}{c}5.9 \\
(5.0)\end{array}$ & $\begin{array}{c}8.4 \\
(7.0)\end{array}$ & $\begin{array}{c}7.5 \\
(7.2)^{*}\end{array}$ & $\begin{array}{c}6.6 \\
(6.5)\end{array}$ & $\begin{array}{c}6.3 \\
(7.0)\end{array}$ \\
\hline \multicolumn{11}{|l|}{ RUB } \\
\hline Low & $\begin{array}{c}6774 \\
(64.8 \%)\end{array}$ & $\begin{array}{c}1227 \\
(67.5 \%)\end{array}$ & $\begin{array}{c}3835 \\
(61.3 \%)\end{array}$ & $\begin{array}{c}531 \\
(55.1 \%)^{*}\end{array}$ & $\begin{array}{c}5555 \\
(33.9 \%)\end{array}$ & $\begin{array}{c}2166 \\
(33.0 \%)\end{array}$ & $\begin{array}{c}14821 \\
(31.9 \%)\end{array}$ & $\begin{array}{c}2864 \\
(29.8 \%)\end{array}$ & $\begin{array}{c}6459 \\
(52.3 \%)\end{array}$ & $\begin{array}{c}1781 \\
(53.1 \%)\end{array}$ \\
\hline Moderate & $\begin{array}{c}2204 \\
(21.1 \%)\end{array}$ & $\begin{array}{c}339 \\
(18.6 \%)\end{array}$ & $\begin{array}{c}1436 \\
(23.0 \%)\end{array}$ & $\begin{array}{c}231 \\
(24.0 \%)\end{array}$ & $\begin{array}{c}6545 \\
(39.9 \%)\end{array}$ & $\begin{array}{c}2533 \\
(38.6 \%)\end{array}$ & $\begin{array}{c}15365 \\
(33.1 \%)\end{array}$ & $\begin{array}{c}3038 \\
(31.7 \%)\end{array}$ & $\begin{array}{c}4393 \\
(35.6 \%)\end{array}$ & $\begin{array}{c}1165 \\
(34.7 \%)\end{array}$ \\
\hline High & $\begin{array}{c}1483 \\
(14.2 \%)\end{array}$ & $\begin{array}{c}253 \\
(13.9 \%)\end{array}$ & $\begin{array}{c}986 \\
(15.8 \%)\end{array}$ & $\begin{array}{c}202 \\
(21.0 \%)^{*}\end{array}$ & $\begin{array}{c}4286 \\
(26.2 \%)\end{array}$ & $\begin{array}{c}1866 \\
(28.4 \%)\end{array}$ & $\begin{array}{c}16282 \\
(35.0 \%)\end{array}$ & $\begin{array}{c}3696 \\
(38.5 \%)\end{array}$ & $\begin{array}{c}1501 \\
(12.2 \%)\end{array}$ & $\begin{array}{c}408 \\
(12.2 \%)\end{array}$ \\
\hline Morbid obesity & $\begin{array}{c}93 \\
(0.9 \%)\end{array}$ & $\begin{array}{c}14 \\
(0.8 \%)\end{array}$ & $\begin{array}{c}39 \\
(0.6 \%)\end{array}$ & $\begin{array}{c}N<6 \\
(<0.6 \%)\end{array}$ & $\begin{array}{c}47 \\
(0.3 \%)\end{array}$ & $\begin{array}{c}38 \\
(0.6 \%)\end{array}$ & $\begin{array}{c}102 \\
(0.2 \%)\end{array}$ & $\begin{array}{c}20 \\
(0.2 \%)\end{array}$ & $\begin{array}{c}310 \\
(2.5 \%)\end{array}$ & $\begin{array}{c}51 \\
(1.5 \%)\end{array}$ \\
\hline \multicolumn{11}{|c|}{ Socio-economic group } \\
\hline $\begin{array}{l}\text { Lowest two } \\
\text { quintiles }\end{array}$ & $\begin{array}{c}4186 \\
(40.0 \%)\end{array}$ & $\begin{array}{c}778 \\
(42.8 \%)\end{array}$ & $\begin{array}{c}2334 \\
(37.3 \%)\end{array}$ & $\begin{array}{c}369 \\
(38.3 \%)\end{array}$ & $\begin{array}{c}5302 \\
(32.4 \%)\end{array}$ & $\begin{array}{c}1789 \\
(27.3 \%)^{*}\end{array}$ & $\begin{array}{c}18003 \\
(38.7 \%)\end{array}$ & $\begin{array}{c}3657 \\
(38.1 \%)\end{array}$ & $\begin{array}{c}4294 \\
(34.8 \%)\end{array}$ & $\begin{array}{c}1127 \\
(33.6 \%)\end{array}$ \\
\hline $\begin{array}{l}\text { Highest two } \\
\text { quintiles }\end{array}$ & $\begin{array}{c}4028 \\
(38.5 \%)\end{array}$ & $\begin{array}{c}678 \\
(37.3 \%)\end{array}$ & $\begin{array}{c}2601 \\
(41.6 \%)\end{array}$ & $\begin{array}{c}372 \\
(38.6 \%)\end{array}$ & $\begin{array}{c}7728 \\
(47.2 \%)\end{array}$ & $\begin{array}{c}3518 \\
(53.6 \%)^{*}\end{array}$ & $\begin{array}{c}18902 \\
(40.7 \%)\end{array}$ & $\begin{array}{c}4161 \\
(43.4 \%)\end{array}$ & $\begin{array}{c}5427 \\
(43.9 \%)\end{array}$ & $\begin{array}{c}1582 \\
(47.2 \%)\end{array}$ \\
\hline $\begin{array}{l}\text { Rural residence } \\
(\%)\end{array}$ & $\begin{array}{c}1289 \\
(12.3 \%)\end{array}$ & $\begin{array}{c}109 \\
(6.0 \%)^{* *}\end{array}$ & $\begin{array}{c}923 \\
(14.8 \%)\end{array}$ & $\begin{array}{c}63 \\
(6.5 \%)^{* *}\end{array}$ & $\begin{array}{c}2597 \\
(15.8 \%)\end{array}$ & $\begin{array}{c}860 \\
(13.1 \%)\end{array}$ & $\begin{array}{c}7214 \\
(15.5 \%)\end{array}$ & $\begin{array}{c}760 \\
(7.9 \%)^{* *}\end{array}$ & $\begin{array}{c}2553 \\
(20.7 \%)\end{array}$ & $\begin{array}{c}322 \\
(9.6 \%)^{* *}\end{array}$ \\
\hline \multicolumn{11}{|l|}{ Anesthetic type } \\
\hline General & $\begin{array}{c}8877 \\
(84.9 \%)\end{array}$ & $\begin{array}{c}1524 \\
(83.8 \%)\end{array}$ & $\begin{array}{c}5182 \\
(82.8 \%)\end{array}$ & $\begin{array}{c}819 \\
(85.0 \%)\end{array}$ & $\begin{array}{c}12761 \\
(77.9 \%)\end{array}$ & $\begin{array}{c}5790 \\
(88.2 \%)^{* *}\end{array}$ & $\begin{array}{c}15888 \\
(34.2 \%)\end{array}$ & $\begin{array}{c}3859 \\
(40.2 \%)^{*}\end{array}$ & $\begin{array}{c}9376 \\
(75.9 \%)\end{array}$ & $\begin{array}{c}1757 \\
(52.4 \%)^{* *}\end{array}$ \\
\hline Epidural/spinal & $\begin{array}{c}737 \\
(7.0 \%)\end{array}$ & $\begin{array}{c}99 \\
(5.4 \%)\end{array}$ & $\begin{array}{c}596 \\
(9.5 \%)\end{array}$ & $\begin{array}{c}75 \\
(7.8 \%)\end{array}$ & $\begin{array}{c}3610 \\
(22.0 \%)\end{array}$ & $\begin{array}{c}764 \\
(11.6 \%)^{* *}\end{array}$ & $\begin{array}{l}29359 \\
(63.2 \%)\end{array}$ & $\begin{array}{c}5422 \\
(56.5 \%)^{*}\end{array}$ & $\begin{array}{c}1593 \\
(12.9 \%)\end{array}$ & $\begin{array}{c}480 \\
(14.3 \%)\end{array}$ \\
\hline Other & $\begin{array}{c}847 \\
(8.1 \%)\end{array}$ & $\begin{array}{c}196 \\
(10.8 \%)\end{array}$ & $\begin{array}{c}479 \\
(7.7 \%)\end{array}$ & $\begin{array}{c}70 \\
(7.3 \%)\end{array}$ & $\begin{array}{c}15 \\
(0.1 \%)\end{array}$ & $\begin{array}{c}11 \\
(0.2 \%)\end{array}$ & $\begin{array}{c}1221 \\
(2.6 \%)\end{array}$ & $\begin{array}{c}317 \\
(3.3 \%)\end{array}$ & $\begin{array}{c}1384 \\
(11.2 \%)\end{array}$ & $\begin{array}{c}1117 \\
(33.3 \%)^{* *}\end{array}$ \\
\hline \multicolumn{11}{|c|}{ Surgeon \& hospital characteristics } \\
\hline Surgeon age & $\begin{array}{l}50.5 \\
(10.8)\end{array}$ & $\begin{array}{c}49.5 \\
(9.8)^{*}\end{array}$ & $\begin{array}{c}48.7 \\
(10.3)\end{array}$ & $\begin{array}{l}47.8 \\
(9.7)\end{array}$ & $\begin{array}{l}46.6 \\
(9.5)\end{array}$ & $\begin{array}{c}47.7 \\
(8.6)^{*}\end{array}$ & $\begin{array}{c}50.2 \\
(10.4)\end{array}$ & $\begin{array}{l}49.7 \\
(9.1)\end{array}$ & $\begin{array}{l}48.4 \\
(8.4)\end{array}$ & $\begin{array}{l}48.8 \\
(8.2)\end{array}$ \\
\hline $\begin{array}{l}\text { Surgeon yearly } \\
\text { volume }\end{array}$ & $\begin{array}{l}10.7 \\
(5.5)\end{array}$ & $\begin{array}{c}8.6 \\
(5.4)^{* *}\end{array}$ & $\begin{array}{c}6.7 \\
(3.4)\end{array}$ & $\begin{array}{c}4.9 \\
(3.0)^{* *}\end{array}$ & $\begin{array}{c}21.5 \\
(12.5)\end{array}$ & $\begin{array}{c}49.3 \\
(35.6)^{* *}\end{array}$ & $\begin{array}{c}41.7 \\
(23.1)\end{array}$ & $\begin{array}{c}31.3 \\
(20.9)^{* *}\end{array}$ & $\begin{array}{c}35.2 \\
(32.6)\end{array}$ & $\begin{array}{c}52.0 \\
(38.6)^{* *}\end{array}$ \\
\hline $\begin{array}{l}\text { Hospital yearly } \\
\text { volume }\end{array}$ & $\begin{array}{c}28.1 \\
(19.9)\end{array}$ & $\begin{array}{c}21.8 \\
(10.6)^{* *}\end{array}$ & $\begin{array}{l}16.1 \\
(9.6)\end{array}$ & $\begin{array}{c}11.5 \\
(5.2)^{* *}\end{array}$ & $\begin{array}{c}51.1 \\
(26.3)\end{array}$ & $\begin{array}{c}121.8 \\
(66.6)^{* *}\end{array}$ & $\begin{array}{l}108.4 \\
(57.0)\end{array}$ & $\begin{array}{l}107.2 \\
(56.3)\end{array}$ & $\begin{array}{c}69.0 \\
(43.1)\end{array}$ & $\begin{array}{c}127.2 \\
(79.4)^{* *}\end{array}$ \\
\hline
\end{tabular}

*Standardized difference between non-teaching and academic hospital for specified procedure is betw
specified procedure is $>0.20 ; n<6$ : Specific number of patients not reported due to privacy regulations. 
Welk et al.

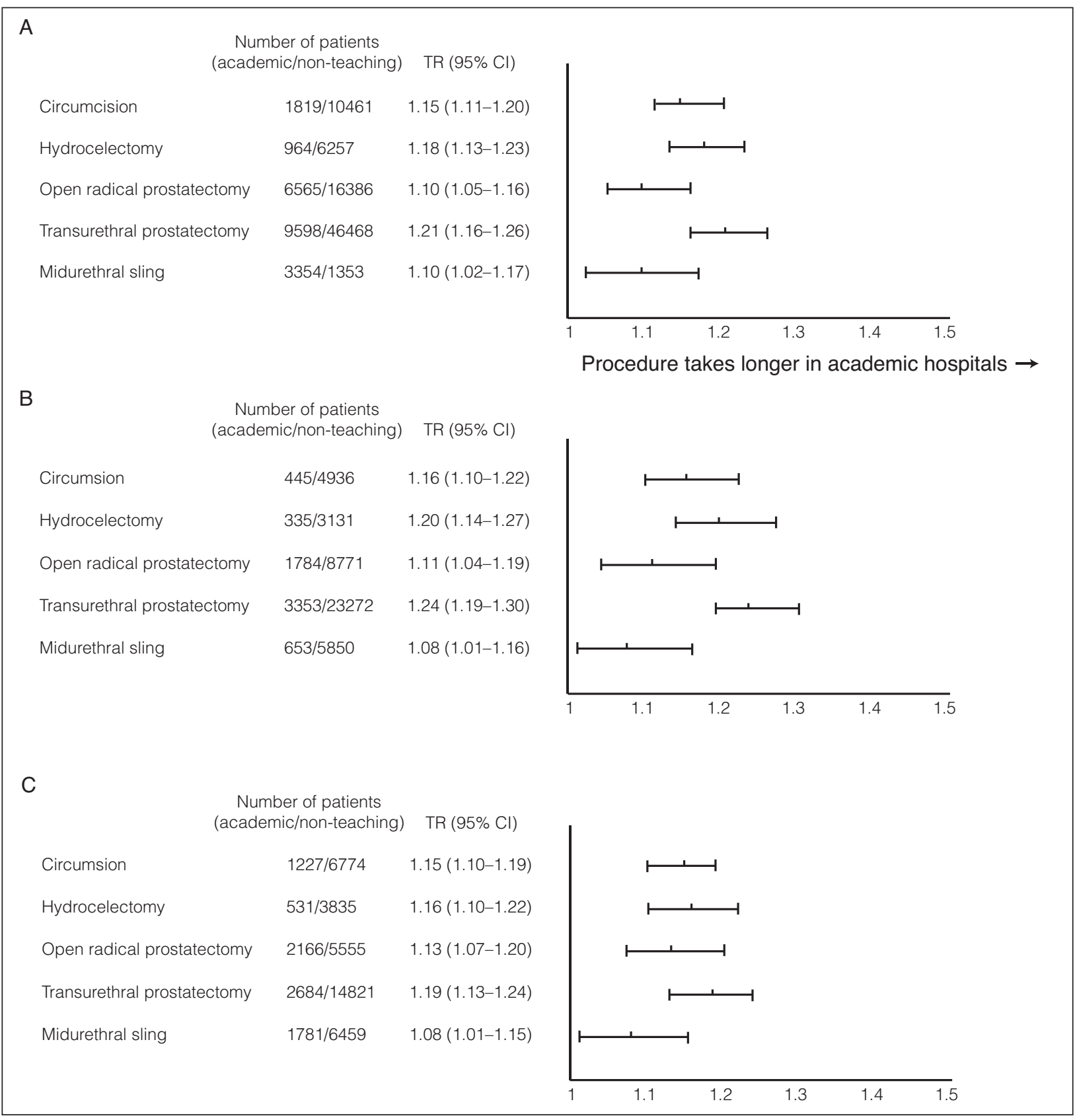

Fig. 2. Forest plot showing time ratio $(95 \% \mathrm{Cl})$ for each of the urological procedures studied. The time ratio (TR) is interpreted as the proportion of extra time required for the procedure in an academic hospital compared to a non-teaching hospital: (A) Primary adjusted analysis; (B) Sensitivity analysis restricted to patients operated on at their expected hospital based on residency; (C) Sensitivity analysis restricted to patients with low comorbidity. The number of patients included from non-teaching hospitals and academic hospitals is included for each procedure.

prolonged operative time observed in academic hospitals across all our procedures persisted (Figs. 2B, C).

\section{Discussion}

We demonstrated that the operative time for common urological procedures is significantly longer in academic centres as opposed to non-teaching hospitals. This difference translated to 8-17 additional minutes for shorter operations (circumcision, hydrocelectomy, transurethral prostatectomy, and mid-urethral sling), or $10-21 \%$ additional operative time. Similarly, a more complex procedure (radical prostatectomy) took an additional 29 minutes, or $10 \%$ longer. As expected, we found that morbid obesity and epidural or spinal anesthetic also significantly prolonged operative procedures. In general, patient comorbidities, surgeon characteristics, and hospital and surgeon volume had only a small magnitude of effect on the duration of the urological 


\begin{tabular}{|c|c|c|c|c|c|}
\hline & Circumcision & Hydrocelectomy & $\begin{array}{l}\text { Open radical } \\
\text { prostatectomy }\end{array}$ & $\begin{array}{l}\text { Transurethral } \\
\text { prostatectomy }\end{array}$ & Mid-urethral sling \\
\hline \multicolumn{6}{|l|}{ Patient characteristics } \\
\hline Age (per 10 years increase) & $1.00(1.00-1.00)$ & $1.00(1.00-1.00)$ & $1.00(1.00-1.00)$ & $1.01(1.01-1.02)^{*}$ & $1.01(1.01-1.01)^{*}$ \\
\hline \multicolumn{6}{|l|}{ RUB (reference=low) } \\
\hline Moderate & $1.00(0.99-1.01)$ & $1.00(0.99-1.01)$ & $1.011 .00-1.01)^{*}$ & $0.98(0.97-0.99)^{*}$ & $1.01(1.01-1.02)^{*}$ \\
\hline High & $1.01(1.00-1.02)^{*}$ & $1.01(1.00-1.03)$ & $1.01(1.00-1.01)^{*}$ & $0.97(0.97-0.98)^{*}$ & $1.03(1.02-1.04)^{*}$ \\
\hline Morbid obesity & $1.07(1.03-1.12)^{*}$ & $1.13(1.05-1.22)^{*}$ & $1.11(1.07-1.15)^{*}$ & $1.09(1.04-1.14)^{*}$ & $1.09(1.07-1.11)^{*}$ \\
\hline \multicolumn{6}{|l|}{ Anesthetic type (reference=GA) } \\
\hline Epidural/spinal & $1.06(1.04-1.08)^{*}$ & $1.07(1.05-1.10)^{*}$ & $1.01(1.00-1.02)$ & $1.01(1.00-1.02)^{*}$ & $1.07(1.05-1.08)^{*}$ \\
\hline Other & $1.01(0.99-1.03)$ & $1.01(0.99-1.03)$ & $0.99(0.95-1.02)$ & $1.01(0.99-1.02)$ & $1.01(1.00-1.03)$ \\
\hline \multicolumn{6}{|l|}{ Surgeon \& hospital characteristics } \\
\hline Surgeon age (per 10-year increase) & $0.98(0.97-0.99)^{*}$ & $0.99(0.98-1.00)$ & $0.98(0.96-1.01)$ & $1.01(0.99-1.02)$ & $0.98(0.96-1.01)$ \\
\hline $\begin{array}{l}\text { Surgeon yearly volume (per } 10 \\
\text { additional procedures) }\end{array}$ & $0.98(0.97-0.99)^{*}$ & $0.97(0.94-0.99)^{*}$ & $0.99(0.99-1.00)^{*}$ & $1.00(0.99-1.00)$ & $1.00(0.99-1.00)$ \\
\hline Hospital volume & $0.99(0.98-1.00)^{*}$ & $0.98(0.97-1.00)^{*}$ & $1.00(1.00-1.01)$ & $1.00(1.00-1.00)$ & $1.00(1.00-1.00)$ \\
\hline $\begin{array}{l}\text { Academic hospital (reference }=\text { non- } \\
\text { teaching hospital) }\end{array}$ & $1.15(1.11-1.20)^{*}$ & $1.18(1.13-1.23)^{*}$ & $1.10(1.05-1.16)^{*}$ & $1.21(1.16-1.26)^{*}$ & $1.10(1.02-1.17)^{*}$ \\
\hline $\begin{array}{l}{ }^{*} \mathrm{p}<0.05 \\
\text { Model was also adjusted for year of cohort entry, a } \\
\text { procedures, the operative duration was significantl }\end{array}$ & 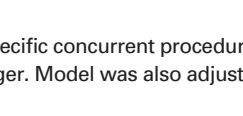 & & & & $\begin{array}{l}\text { urrent } \\
\text { small number }\end{array}$ \\
\hline
\end{tabular}

procedures we assessed. Assigning an average value of $\$ 10$ per minute of operating time (which accounts for the fixed, non-consumable costs, such as nursing salary, utilities, and equipment depreciation ${ }^{8}$ ), the additional cost of carrying out these five procedures in our select cohort during the study period (above that required if they were done in non-teaching hospitals) was $\$ 4.25$ million dollars. This is significant in a publically funded healthcare system, where the use of operating room time is $>95 \%$ and tax payers cover the costs of any inefficiencies in the operating room.

Our results are generally consistent with the existing literature on resident involvement and operative times. The majority of the prior literature is based on data from the National Surgical Quality Improvement Program (NSQIP) in the U.S. The involvement of residents (including junior, senior, and chief residents) significantly increased the risk of an operative time $>75^{\text {th }}$ percentile for minimally invasive partial and radical nephrectomies, as well minimally invasive radical prostatectomy. ${ }^{4}$ Similarly, there was a twofold higher chance of a prolonged operative time (defined as $>75 \%$ percentile) when urology residents were involved with transurethral surgery. ${ }^{5}$ Among basic general surgery procedures (laparoscopic cholecystectomy or appendectomy, or open inguinal hernia repair, all of which tended to take approximately an hour), skin-to-skin operative time increased by 12-20 minutes with the involvement of both junior or senior residents, compared to the attending surgeon operating alone. ${ }^{7}$

It is essential for academic urologists and anesthesiologist to continue to balance the priorities of resident education and hands-on learning opportunities with the efficient and responsible use of publicly funded healthcare resources, such as operating room time. In addition to resource implications, previous studies have demonstrated an increased risk in complications, such as surgical site infections, among certain surgical procedures when they are significantly prolonged. ${ }^{17}$ This potential risk is balanced by studies suggesting that, in general, patients do not have worse outcomes if residents are involved in their surgical procedure. ${ }^{18}$ The unique responsibilities and contributions of teaching hospitals should be recognized by funding bodies and activitybased funding should account for the increased operative time associated with training the next generation of medical professionals.

Our study has several unique features that add to the existing literature. First, the operations we studied were chosen due to their infrequent need to refer to a tertiary care academic centre. It is likely that some of the previously studied procedures were prone to a significant referral bias and cases with increased complexity would not be adequately captured within administrative data. Our two sensitivity analyses, which attempt to adjust for referral bias, demonstrated results consistent with our primary analysis. Second, we captured the entire duration of the operative process. While a comparable operative speed may be achieved by a surgeon with and without a trainee, the effect of other components of the academic hospital operating room (such as anesthesia residents or operating room nurses in training) also need to be taken into account, as ultimately this time is attributed to the surgeon's procedure. Third, we adjusted 
Welk et al.

our estimates for several potential variables outside of patient comorbidities, such as surgeon experience and hospital volumes. Finally, these results are from a publically funded healthcare system, which may be subject to different financial pressures and potential inefficiencies than the NSQIP hospitals.

The limitations of our study also need to be acknowledged. We could not actually determine which specific trainees were involved with individual cases. This concern is minimized by a long-standing priority among all urology residency programs to ensure operative cases are attended by a resident or fellow and, if anything, the lack of trainee participation in an academic centre should bias the time ratios towards 1.0. This also means it is impossible to separate out the effects of medical students, nurses in training, anesthesiologists, urologists, and general systematic differences in preoperative, operative, and postoperative care and patient flow that is inherent in large academic hospitals. Our results are based on the single payer, publically funded Canadian healthcare system, and may not be generalizable to hospitals that use different funding models. Misclassification or residual confounding is always a possibility with an observational study and, despite the fact the procedures are generally straightforward, it is possible there were unadjusted systematic differences between patients being treated at academic vs. non-teaching centres.

\section{Conclusion}

A variety of general urological surgical procedures take $10-21 \%$ longer to complete in academic hospitals as opposed to non-teaching hospitals. It is likely that the involvement of various healthcare trainees plays a significant role in this effect and it should be taken into account when developing activity-based funding models.

Competing interests: Dr. Welk has received grant funding from Astellas. The remaining authors declare no competing personal or financial interests.

Acknowledgements: We would like to thank Drs. Amit Garg, David Urbach, and Salimah Shariff for their support and input throughout the study process. This study was funded in part by the Academic Medical Organization of Southwestern Ontario (AMOSO) AHSC AFP Innovation Fund and the London Health Sciences Centre Department of Surgery. This study was supported by the ICES Western site. ICES is funded by an annual grant from the Ontario Ministry of Health and Long-Term Care (MOHLTC). Core funding for ICES Western is provided by AMOSO, the Schulich School of Medicine and Dentistry (SSMD), Western University, and the Lawson Health Research Institute (LHRI). The opinions, results, and conclusions reported in this paper are those of the authors and are independent from the funding sources. No endorsement by ICES, AMOSO, SSMD, LHRI, or the Ontario MOHLTC is intended or should be inferred. Parts of this material are based on data and information compiled and provided by $\mathrm{CIHI}$. However, the analyses, conclusions, opinions, and statements expressed herein are those of the authors, and not necessarily those of $\mathrm{CIHI}$.

\section{References}

1. Ideas and opportunities for bending the healthcare cost curve. $2010 \mathrm{Apr}$ 13; https://www.oha.com/ KnowledgeCentre/Library/Documents/Bending\%20the\%20Health\%20Care $\% 20$ Cost $\% 20$ Curve $\% 20$ (Final\%20Report\%20\%20April\%2013\%202010).pdf. Accessed February 1, 2016.

2. Integrated funding. 2015 May 26; hitp://healthcarefunding2.sites.olt.ubc.ca/files/2015/06/WhitePaper-Integrated-Funding.pdf. Accessed January 20, 2016.

3. Excellent care for all - Healthcare Professionals - MOHLTC. 2016. http://www.health.gov.on.ca/en/ pro/programs/ecfa/funding/hs_funding_abp.aspx. Accessed Feb 14, 2016.

4. Ruhotina $N$, Dagenais J, Gandaglia $G$, et al. The impact of resident involvement in minimally invasive urologic oncology procedures. Can Urol Assoc J 2014;8:334-40. http://dx.doi.org/10.5489/cuaj.2170

5. Allard $C B$, Meyer $C P$, Gandaglia $G$, et al. The effect of resident involvement on perioperative outcomes in transurethral urologic surgeries. J Surg Educ 2015;72:1018-25. http://dx.doi.org/10.1016/i. jsurg.2015.04.012

6. Igwe E, Hernandez E, Rose $\mathrm{S}$, et al. Resident participation in laparoscopic hysterectomy: Impact of trainee involvement on operative times and surgical outcomes. Am J Obstet Gynecol 2014;211:484.el-7. http:// dx.doi.org/10.1016/i.ajog.2014.06.024

7. Papandria $D$, Rhee $D$, Ortega $G$, et al. Assessing trainee impact on operative time for common general surgical procedures in ACS-NSQIP. J Surg Educ 2012;69:149-55. http://dx.doi.org/10.1016/i. jsurg.2011.08.003

8. Goldstein L, Ondrejicka DA. A literature review and micro-costing approach to determine the cost of one hour of operating time in Canada. Value in Health 2015;18:A83. http://dx.doi.org/10.1016/i. jval.2015.03.486

9. Jha P, Deboer D, Sykora K, et al. Characteristics and mortality outcomes of thrombolysis trial participants and nonparticipants: A population-based comparison. J Am Coll Cardiol 1996;27:1335-42. http://dx.doi. org/10.1016/0735-1097(96)00018-6

10. Williams J, Young W. Appendix I: A summary of studies on the quality of health care administrative databases in Canada. In: Goel V, Williams JI, Anderson GM, et al, eds. Patterns of Health Care in Ontario: the ICES Practice Atlas. 2nd ed. Ottawa, ON: Canadian Medical Association; 2000:339-347.

11. Raina $P$, Torrance-Rynard V, Wong $M$, et al. Agreement between self-reported and routinely collected healthcare utilization data among seniors. Health Serv Res 2002;37:751-74. http://dx.doi. org/10.1111/1475-6773.00047

12. Redelmeier DA, Thiruchelvam D, Daneman N. Introducing a methodology for estimating duration of surgery in health services research. J Clin Epidemiol 2008;61:882-9. http://dx.doi.org/10.1016/i. jclinepi.2007.10.015

13. Kralji B. Measuring "rurality" for purposes of health care planning: An emperical measure for Ontario. Ontario Medical Review 2000:33-52.

14. Austin PC, van Walraven C, Wodchis WP, et al. Using the Johns Hopkins Aggregated Diagnosis Groups (ADGs) to predict mortality in a general adult population cohort in Ontario, Canada. Med Care 2011;49:932-9. http://dx.doi.org/10.1097/MLR.0b013e318215d5e2

15. Austin $\mathrm{PC}$. Using the standardized difference to compare the prevalence of a binary variable between two groups in observational research. Commun Stat Simul Comput 2009;38:1228-34. http://dx.doi. org/10.1080/03610910902859574

16. Stukel TA, Glazier RH, Schultz SE, et al. Multispecialty physician networks in Ontario. Open Med 2013;7:e40-55

17. Leong G, Wilson J, Charlett A. Duration of operation as a risk factor for surgical site infection: Comparison of English and US data. J Hosp Infect 2006;63:255-62. http://dx.doi.org/10.1016/i.hin.2006.02.007

18. Saliba AN, Taher AT, Tamim H, et al. Impact of resident involvement in surgery (IRIS-NSQIP): Looking at the bigger picture based on the American College of Surgeons-NSQIP database. J Am Coll Surg 2016;222:30-40. http://dx.doi.org/10.1016/i.jamcollsurg.2015.10.011

Correspondence: Dr. Blayne Welk, University of Western Ontario, London, ON, Canada; bkwelk@gmail.com 
Welk et al.

\section{eTable 1. STROBE checklist for cohort studies}

\begin{tabular}{|c|c|c|c|}
\hline & $\begin{array}{l}\text { Item } \\
\text { No. }\end{array}$ & Recommendation & Page no. \\
\hline \multirow{2}{*}{$\begin{array}{l}\text { Title and } \\
\text { abstract }\end{array}$} & & (a) Indicate the study's design with a commonly used term in the title or the abstract & Abstract, methods \\
\hline & 1 & $\begin{array}{l}\text { (b) Provide in the abstract an informative and balanced summary of what was done and } \\
\text { what was found }\end{array}$ & Abstract \\
\hline \multicolumn{4}{|l|}{ Introduction } \\
\hline $\begin{array}{l}\text { Background/ } \\
\text { rationale }\end{array}$ & 2 & Explain the scientific background and rationale for the investigation being reported & Introduction \\
\hline Objectives & 3 & State specific objectives, including any prespecified hypotheses & $\begin{array}{l}\text { Methods, primary } \\
\text { outcome and } \\
\text { exposure }\end{array}$ \\
\hline \multicolumn{4}{|l|}{ Methods } \\
\hline Study design & 4 & Present key elements of study design early in the paper & $\begin{array}{l}\text { Methods, study design } \\
\text { and setting }\end{array}$ \\
\hline Setting & 5 & $\begin{array}{l}\text { Describe the setting, locations, and relevant dates, including periods of recruitment, } \\
\text { exposure, follow-up, and data collection }\end{array}$ & $\begin{array}{l}\text { Methods, study design } \\
\text { and setting, primary } \\
\text { exposure }\end{array}$ \\
\hline Participants & 6 & $\begin{array}{l}\text { (a) Cohort study - Give the eligibility criteria, and the sources and methods of selection } \\
\text { of participants. Describe methods of followup }\end{array}$ & Methods \\
\hline Variables & 7 & $\begin{array}{l}\text { Clearly define all outcomes, exposures, predictors, potential confounders, and effect } \\
\text { modifiers. Give diagnostic criteria, if applicable }\end{array}$ & $\begin{array}{l}\text { Methods, primary } \\
\text { outcome and } \\
\text { exposure, eTable } 2\end{array}$ \\
\hline $\begin{array}{l}\text { Data sources/ } \\
\text { measurement }\end{array}$ & 8 & $\begin{array}{l}\text { For each variable of interest, give sources of data and details of methods of assessment } \\
\text { (measurement). Describe comparability of assessment methods if there is more than one } \\
\text { group }\end{array}$ & $\begin{array}{l}\text { Methods, data } \\
\text { sources, eTable } 2\end{array}$ \\
\hline Bias & 9 & Describe any efforts to address potential sources of bias & $\begin{array}{l}\text { Methods, statistical } \\
\text { analysis }\end{array}$ \\
\hline Study size & 10 & Explain how the study size was arrived at & $\begin{array}{l}\text { Methods, (population- } \\
\text { based study) }\end{array}$ \\
\hline $\begin{array}{l}\text { Quantitative } \\
\text { variables }\end{array}$ & 11 & $\begin{array}{l}\text { Explain how quantitative variables were handled in the analyses. If applicable, describe } \\
\text { which groupings were chosen and why }\end{array}$ & $\begin{array}{l}\text { Methods, primary } \\
\text { outcome and } \\
\text { exposure, covariates }\end{array}$ \\
\hline \multirow{5}{*}{$\begin{array}{l}\text { Statistical } \\
\text { methods }\end{array}$} & \multirow{5}{*}{12} & (a) Describe all statistical methods, including those used to control for confounding & $\begin{array}{l}\text { Methods, statistical } \\
\text { analysis }\end{array}$ \\
\hline & & (b) Describe any methods used to examine subgroups and interactions & None \\
\hline & & (c) Explain how missing data were addressed & Methods \\
\hline & & (d) Cohort study-If applicable, explain how loss to follow-up was addressed & NA \\
\hline & & (e) Describe any sensitivity analyses & $\begin{array}{l}\text { Methods, statistical } \\
\text { analysis }\end{array}$ \\
\hline
\end{tabular}


eTable 1 (cont'd). STROBE checklist for cohort studies

\begin{tabular}{|c|c|c|c|}
\hline & $\begin{array}{l}\text { Item } \\
\text { No. }\end{array}$ & Recommendation & Page no. \\
\hline \multicolumn{4}{|c|}{ Methods (cont'd) } \\
\hline \multirow[t]{3}{*}{ Participants } & \multirow[t]{3}{*}{13} & $\begin{array}{l}\text { (a) Report numbers of individuals at each stage of study - e.g., numbers potentially } \\
\text { eligible, examined for eligibility, confirmed eligible, included in the study, completing } \\
\text { follow-up, and analysed }\end{array}$ & Methods \\
\hline & & (b) Give reasons for non-participation at each stage & Methods \\
\hline & & (c) Consider use of a flow diagram & \\
\hline \multirow{3}{*}{$\begin{array}{l}\text { Descriptive } \\
\text { data }\end{array}$} & \multirow{3}{*}{14} & $\begin{array}{l}\text { (a) Give characteristics of study participants (e.g. demographic, clinical, social) and } \\
\text { information on exposures and potential confounders }\end{array}$ & Results, Table 1 \\
\hline & & (b) Indicate number of participants with missing data for each variable of interest & NA \\
\hline & & (c) Cohort study - Summarize followup time (e.g., average and total amount) & NA \\
\hline Outcome data & 15 & Cohort study - Report numbers of outcome events or summary measures over time & NA \\
\hline \multirow{3}{*}{ Main results } & \multirow{3}{*}{16} & $\begin{array}{l}\text { (a) Give unadjusted estimates and, if applicable, confounder-adjusted estimates and their } \\
\text { precision (e.g., 95\% confidence interval). Make clear which confounders were adjusted for } \\
\text { and why they were included }\end{array}$ & Results, Table 2 \\
\hline & & (b) Report category boundaries when continuous variables were categorized & $\begin{array}{l}\text { Results } \\
\text { standard deviation }\end{array}$ \\
\hline & & $\begin{array}{l}\text { (c) If relevant, consider translating estimates of relative risk into absolute risk for a } \\
\text { meaningful time period }\end{array}$ & $\begin{array}{l}\text { Results } \\
\text { Time ratios (analogous } \\
\text { to relative risk) }\end{array}$ \\
\hline Other analyses & 17 & $\begin{array}{l}\text { Report other analyses done - e.g., analyses of subgroups and interactions, and } \\
\text { sensitivity analyses }\end{array}$ & Figs. 2B, C \\
\hline \multicolumn{4}{|c|}{ e } \\
\hline Key results & 18 & Summarize key results with reference to study objectives & Discussion \\
\hline Limitations & 19 & $\begin{array}{l}\text { Discuss limitations of the study, taking into account sources of potential bias or } \\
\text { imprecision. Discuss both direction and magnitude of any potential bias }\end{array}$ & Discussion \\
\hline Interpretation & 20 & $\begin{array}{l}\text { Give a cautious overall interpretation of results considering objectives, limitations, } \\
\text { multiplicity of analyses, results from similar studies, and other relevant evidence }\end{array}$ & Discussion \\
\hline Generalizability & 21 & Discuss the generalisability (external validity) of the study results & Discussion \\
\hline \multicolumn{4}{|c|}{ Other information } \\
\hline Funding & 22 & $\begin{array}{l}\text { Give the source of funding and the role of the funders for the present study and, if } \\
\text { applicable, for the original study on which the present article is based }\end{array}$ & Acknowledgements \\
\hline
\end{tabular}


Welk et al.

eTable 2. Coding elements used to identify the specified urologic procedures. Both a physician billing code (OHIP) and a matching hospital procedure code (CIHI-DAD/SDS) had to be present

\begin{tabular}{|c|c|c|}
\hline Urology procedure & OHIP code & $\mathrm{CCl}$ code \\
\hline \multirow[t]{5}{*}{ Midurethral sling } & S815 & 1.PL.74.CR-XX-N \\
\hline & & 1.PL.74.AL-XX-N \\
\hline & & 1.PL.74.AF-XX-N \\
\hline & & 1.PL.74.LA-XX-N \\
\hline & & 1.PL.74.DA-XX-N \\
\hline \multirow[t]{6}{*}{$\begin{array}{l}\text { Transurethral } \\
\text { prostatectomy }\end{array}$} & S655 & 1.QT.59.BA-GX \\
\hline & & 1.QT.59.BA-AG \\
\hline & & 1.0T.87.BA \\
\hline & & 1.QT.87.BA-AG \\
\hline & & 1.QT.87.BA-AK \\
\hline & & 1.QT.87.BA-GX \\
\hline \multirow[t]{3}{*}{ Hydrocelectomy } & S611 & 1.QH.80.LA \\
\hline & & 1.QH.87.LA \\
\hline & & 1.QH.87.LB \\
\hline \multirow[t]{4}{*}{ Circumcision } & S573 & 1.QD.89.LA \\
\hline & & 1.QD.89.LA-FF \\
\hline & & 1.QD.72.LA \\
\hline & & 1.QD.89.WJ \\
\hline \multirow[t]{2}{*}{$\begin{array}{l}\text { Open radical } \\
\text { prostatectomy }\end{array}$} & S651 & 1.QT.91.PB \\
\hline & & 1.QT.91.PK \\
\hline
\end{tabular}

CCl: Canadian Classification of Health Intervention; CIHI-DAD/SDS: Canadian Institute for Health Information's Discharge Abstract Database and Same Day Surgery; OHIP: Ontario Health Insurance Plan.

\begin{tabular}{|c|c|c|c|c|c|c|c|c|c|c|c|c|}
\hline Procedure & 2002 & 2003 & 2004 & 2005 & 2006 & 2007 & 2008 & 2009 & 2010 & 2011 & 2012 & Total \\
\hline Circumcision & 1035 & 1081 & 1083 & 1028 & 1039 & 1042 & 1115 & 1297 & 1210 & 1130 & 1220 & 12280 \\
\hline Hydrocelectomy & 653 & 612 & 644 & 625 & 627 & 626 & 658 & 660 & 682 & 695 & 739 & 7221 \\
\hline $\begin{array}{l}\text { Open radical } \\
\text { prostatectomy }\end{array}$ & 1944 & 1859 & 2095 & 2327 & 2239 & 2322 & 2124 & 2098 & 2120 & 2170 & 1653 & 22951 \\
\hline $\begin{array}{l}\text { Transurethral } \\
\text { prostatectomy }\end{array}$ & 4787 & 4835 & 5327 & 5420 & 4993 & 4883 & 5083 & 5145 & 5013 & 5183 & 5397 & 56066 \\
\hline Mid-urethral sling & 0 & 0 & 0 & 0 & 0 & 319 & 2433 & 3123 & 3219 & 3589 & 3024 & 15707 \\
\hline
\end{tabular}

eTable 4. Frequency of concurrent procedures (non-teaching versus academic), and the adjusted time ratio (TR) with $95 \%$ confidence interval

\begin{tabular}{|c|c|c|c|}
\hline Index procedure & Concurrent procedure & $\begin{array}{l}\text { Frequency (Non-teaching } \\
\text { vs. academic) }\end{array}$ & $\begin{array}{l}\text { Adjusted time ratio as a result of } \\
\text { the concurrent procedure }\end{array}$ \\
\hline Circumcision & Chordee/Peyronie's repair & $5.6 \%$ vs. $3.3 \% *$ & $1.08(1.05-1.11)$ \\
\hline Hydrocelectomy & Concurrent spermatocele repair & $24.9 \%$ vs. $10.5 \% * *$ & $1.06(1.04-1.07)$ \\
\hline Open radical prostatectomy & Pelvic lymphadenectomy & $91.2 \%$ vs. $87.9 \% *$ & $1.06(1.03-1.08)$ \\
\hline \multirow[t]{3}{*}{ Transurethral prostatectomy } & Removal of bladder stone & $1.5 \%$ vs. $1.1 \%$ & $1.09(1.07-1.12)$ \\
\hline & Cystolithopaxy & $6.3 \%$ vs. $6.8 \%$ & $1.22(1.20-1.23)$ \\
\hline & Prostate biopsy & $2.3 \%$ vs. $1.6 \% *$ & $1.09(1.06-1.12)$ \\
\hline \multirow[t]{4}{*}{ Mid-urethral sling } & Anterior or posterior repair & $8.6 \%$ vs. $11.5 \% *$ & $1.30(1.26-1.34)$ \\
\hline & Anterior and posterior repair & $3.1 \%$ vs. $4.4 \%$ & $1.60(1.56-1.64)$ \\
\hline & Repair of enterocele & $1.9 \%$ vs. $6.7 \% * *$ & $1.76(1.70-1.81)$ \\
\hline & Hysterectomy with prolapse repair & $1.5 \%$ vs. $3.8 \% *$ & $2.44(2.31-2.59)$ \\
\hline
\end{tabular}

*Standardized difference between non-teaching and academic hospital for specified procedure is $0.10-0.20 ;{ }^{* *}$ Standardized difference between non-teaching and academic hospital for specified procedure is $>0.20$. 